\title{
Symmetry Classification of the One-Dimensional Second Order Equation of a Hydrodynamic Type
}

\author{
Vyacheslav BOYKO \\ Institute of Mathematics of the National Academy of Sciences of Ukraïna, \\ 3 Tereshchenkivs'ka Street, Kyïv 4, Ukraïna
}

\begin{abstract}
The paper contains a symmetry classification of the one-dimensional second order equation of a hydrodynamical type $L(L u)+\lambda L u=F(u)$, where $L \equiv \partial_{t}+u \partial_{x}$. Some classes of exact solutions of this equation are given.
\end{abstract}

In $[1,2]$, the following generalized Navier-Stokes equation

$$
\lambda_{1} L \vec{v}+\lambda_{2} L(L \vec{v})=F\left(\vec{v}^{2}\right) \vec{v}+\lambda_{4} \nabla p
$$

was proposed, where

$$
L \equiv \frac{\partial}{\partial t}+v^{l} \frac{\partial}{\partial x_{l}}+\lambda_{3} \triangle, \quad l=1,2,3,
$$

$\vec{v}=\left(v^{1}, v^{2}, v^{3}\right), v^{l}=v^{l}(t, \vec{x}), p=p(t, \vec{x}), \nabla$ is the gradient, $\triangle$ is the Laplace operator, $\lambda_{1}, \lambda_{2}, \lambda_{3}, \lambda_{4}$ are arbitrary real parameters, $F\left(\vec{v}^{2}\right)$ is an arbitrary differentiable function.

In the one-dimensional scalar case, when $\lambda_{3}=0, \lambda_{4}=0$, Eq.(1) has the form

$$
\lambda_{1} L u+\lambda_{2} L(L u)=F(u),
$$

where $u=u(t, x), L \equiv \partial_{t}+u \partial_{x}$.

In the case when $\lambda_{2}=0$ and $F(u)=0$, Eq.(2) is known to describe the simple wave

$$
u=\varphi(x-t u),
$$

where $\varphi$ is an arbitrary function.

If $\lambda_{2} \neq 0$, then Eq.(2) can be rewritten in the form

$$
L(L u)+\lambda L u=F(u), \quad \lambda=\text { const. }
$$

Eq.(4) in an expanded form is written as follows

$$
\frac{\partial^{2} u}{\partial t^{2}}+2 u \frac{\partial^{2} u}{\partial t \partial x}+\frac{\partial u}{\partial t} \frac{\partial u}{\partial x}+u\left(\frac{\partial u}{\partial x}\right)^{2}+u^{2} \frac{\partial^{2} u}{\partial x^{2}}+\lambda\left(\frac{\partial u}{\partial t}+u \frac{\partial u}{\partial x}\right)=F(u) .
$$

This equation with arbitrary $F(u)$ is evidently invariant under the two-dimensional algebra of translations that is determined by the operators

$$
P_{0}=\partial_{t}, \quad P_{1}=\partial_{x} .
$$


In the present paper, we carry out a symmetry classification of Eq.(4), i.e., we describe functions $F(u)$, with which Eq.(4) admits more extensive Lie algebras than the twodimensional algebra of translations (5). A symmetry classification of (4) is performed using the Lie algorithm $[4,5,6]$ in the class of first-order differential operators

$$
X=\xi^{0}(t, x, u) \partial_{t}+\xi^{1}(t, x, u) \partial_{x}+\eta(t, x, u) \partial_{u} .
$$

Remark. In cases the $\mathbf{1 . 4}, \mathbf{2 . 3}, \mathbf{2 . 4}$, we assume that

$$
\frac{\partial \xi^{0}}{\partial u}=0, \quad \frac{\partial \xi^{1}}{\partial u}=0
$$

It is obvious that the cases $\lambda=0$ and $\lambda \neq 0$ will be essentially different for the investigation of symmetries of Eq.(4). If $\lambda \neq 0$, then one can always set $\lambda \equiv 1$ (there exists such a change of variables). For this reason, we consider the cases $\lambda=0$ and $\lambda=1$ separately.

I. Let us consider Eq.(4), when $\lambda=0$, i.e., the equation

$$
L(L u)=F(u) .
$$

Symmetry classification of (7) leads to five distinct cases.

Case 1.1. $F(u)$ is an arbitrary continuously differentiable function.

The maximal invariance algebra in this case is the two-dimensional algebra (5).

Case 1.2. $\quad F(u)=a \exp (u), \quad a, b$ - const, $a \neq 0, b \neq 0$.

The maximal invariance algebra of the equation

$$
L(L u)=a \exp (u)
$$

is a three-dimensional algebra $\left\langle P_{0}, P_{1}, Y\right\rangle$, where

$$
Y=t \partial_{t}+(x-2 t) \partial_{x}-2 \partial_{u} .
$$

We note that $Y$ can be represented as the linear combination of the dilation and Galilei operators

$$
Y=\left(t \partial_{t}+x \partial_{x}\right)-2\left(t \partial_{x}+\partial_{u}\right)=D-2 G .
$$

The operators $D$ and $G$ commute, thus the transformations corresponding to $Y$ can be interpreted as a composition of dilation and Galilei transformations, i.e., as a composition of a dilation in $t$ and $x$ with a change of the inertial system. On the other hand, the operators $\left\langle P_{0}, P_{1}, Y>\right.$ form a subalgebra of the extended Galilei algebra, although the extended Galilei algebra is not an invariance algebra of Eq.(8). The same results are valid for other cases of Eq.(4).

Case 1.3. $F(u)=a(u+b)^{p}, \quad a, b, p-$ const, $a \neq 0, p \neq 0, p \neq 1$.

The maximal invariance algebra of the equation

$$
L(L u)=a(u+b)^{p}
$$

is a three-dimensional algebra $\left\langle P_{0}, P_{1}, R\right\rangle$, where

$$
R=t \partial_{t}+\left(\frac{p-3}{p-1} x-\frac{2 b}{p-1} t\right) \partial_{x}-\frac{2}{p-1}(u+b) \partial_{u} .
$$


Case 1.4. $F(u)=a u+b, \quad a, b-$ const, $a \neq 0$

Performing a change of variables, one can always set $a \equiv 1$ or $a \equiv-1$. Let us consider these cases.

a) The invariance algebra of the equation

$$
L(L u)=u+b
$$

is a seven-dimensional algebra $<P_{0}, P_{1}, Y_{1}, Y_{2}, Y_{3}, Y_{4}, Y_{5}>$, where

$$
\begin{aligned}
& Y_{1}=(x+b t) \partial_{x}+(u+b) \partial_{u}, \\
& Y_{2}=\cosh t \partial_{x}+\sinh t \partial_{u}, \\
& Y_{3}=\sinh t \partial_{x}+\cosh t \partial_{u}, \\
& Y_{4}=\cosh t \partial_{t}+(x+b t) \sinh t \partial_{x}+((x+b t) \cosh t+b \sinh t) \partial_{u} \\
& Y_{5}=\sinh t \partial_{t}+(x+b t) \cosh t \partial_{x}+((x+b t) \sinh t+b \cosh t) \partial_{u} .
\end{aligned}
$$

b) The invariance algebra of the equation

$$
L(L u)=-u+b
$$

is a seven-dimensional algebra $<P_{0}, P_{1}, R_{1}, R_{2}, R_{3}, R_{4}, R_{5}>$, where

$$
\begin{aligned}
& R_{1}=(x-b t) \partial_{x}+(u-b) \partial_{u}, \\
& R_{2}=\cos t \partial_{x}-\sin t \partial_{u}, \\
& R_{3}=\sin t \partial_{x}+\cos t \partial_{u}, \\
& R_{4}=-\cos t \partial_{t}+(x-b t) \sin t \partial_{x}+((x-b t) \cos t-b \sin t) \partial_{u}, \\
& R_{5}=\sin t \partial_{t}+(x-b t) \cos t \partial_{x}-((x-b t) \sin t+b \cos t) \partial_{u} .
\end{aligned}
$$

Case 1.5. $F(u)=a, \quad a=$ const.

In the case $a \neq 0$, there exists a change of variables, so that without loss of generality we can assume that $a \equiv 1$. Thus we consider the cases $a=0$ and $a=1$ separately.

a) The maximal invariance algebra of the equation

$$
L(L u)=0
$$

is a ten-dimensional algebra $<P_{0}, P_{1}, G, D, D_{1}, A_{1}, A_{2}, A_{3}, A_{4}, A_{5}>$,

$$
\begin{aligned}
& G=t \partial_{x}+\partial_{u} \\
& D=t \partial_{t}+x \partial_{x} \\
& D_{1}=x \partial_{x}+u \partial_{u} \\
& A_{1}=\frac{1}{2} t^{2} \partial_{t}+t x \partial_{x}+x \partial_{u}, \\
& A_{2}=\frac{1}{2} t^{2} \partial_{x}+t \partial_{u} \\
& A_{3}=u \partial_{t}+\frac{1}{2} u^{2} \partial_{x} \\
& A_{4}=(t u-x) \partial_{t}+\frac{1}{2} t u^{2} \partial_{x}+\frac{1}{2} u^{2} \partial_{u}, \\
& A_{5}=\left(t^{2} u-2 t x\right) \partial_{t}+\left(\frac{1}{2} t^{2} u^{2}-2 x^{2}\right) \partial_{x}+\left(t u^{2}-2 x u\right) \partial_{u} .
\end{aligned}
$$


Note, that the subalgebras $\left\langle P_{0}, P_{1}, G\right\rangle$ and $\left\langle A_{1},-A_{2}, G\right\rangle$ define two different representations of the Galilei algebra $A G(1,1)$ [4].

b) The maximal invariance algebra of the equation

$$
L(L u)=1
$$

is a ten-dimensional algebra $<P_{0}, P_{1}, G, B_{1}, B_{2}, B_{3}, A_{2}, B_{4}, B_{5}, B_{6}>$,

$$
\begin{aligned}
& B_{1}=t \partial_{t}+3 x \partial_{x}+2 u \partial_{u} \\
& B_{2}=\left(x-\frac{1}{6} t^{3}\right) \partial_{x}+\left(u-\frac{1}{2} t^{2}\right) \partial_{u} \\
& B_{3}=\frac{1}{2} t^{2} \partial_{t}+\left(t x+\frac{1}{12} t^{4}\right) \partial_{x}+\left(x+\frac{1}{3} t^{3}\right) \partial_{u} \\
& B_{4}=\left(u-\frac{1}{2} t^{2}\right) \partial_{t}+\left(\frac{1}{2} u^{2}-\frac{1}{8} t^{4}\right) \partial_{x}+\left(t u-\frac{1}{2} t^{3}\right) \partial_{u} \\
& B_{5}=\left(t u-x-\frac{1}{3} t^{3}\right) \partial_{t}+\left(\frac{1}{2} t u^{2}-\frac{1}{2} t^{2} x-\frac{1}{24} t^{5}\right) \partial_{x}+ \\
& \quad\left(\frac{1}{2} u^{2}+\frac{1}{2} t^{2} u-t x-\frac{5}{24} t^{4}\right) \partial_{u} \\
& B_{6}=\left(t^{2} u-2 t x-\frac{1}{6} t^{4}\right) \partial_{t}+\left(\frac{1}{2} t^{2} u^{2}-2 x^{2}-\frac{1}{3} t^{3} x-\frac{1}{72} t^{6}\right) \partial_{x}+ \\
& \quad\left(t u^{2}-2 x u+\frac{1}{3} t^{3} u-t^{2} x-\frac{1}{12} t^{5}\right) \partial_{u} .
\end{aligned}
$$

Note, that this algebra includes also two different Galilei algebras $\left\langle P_{0}, P_{1}, G\right\rangle$ and $<B_{3},-A_{2}, G>$ as subalgebras.

II. Let us consider Eq.(4) for $\lambda \neq 0$. As was noticed above, we can set $\lambda \equiv 1$. Symmetry classification gives in this case four essentially distinct cases.

Case 2.1. $F(u)$ is an arbitrary continuously differentiable function. The maximal invariance algebra of the equation

$$
L(L u)+L u=F(u),
$$

is the two-dimensional algebra (5).

Case 2.2. $\quad F(u)=a u^{3}-\frac{2}{9} u, \quad a=$ const, $a \neq 0$.

The maximal invariance algebra of the equation

$$
L(L u)+L u=a u^{3}-\frac{2}{9} u
$$

is a three-dimensional algebra $\left\langle P_{0}, P_{1}, Z>\right.$, where

$$
Z=\exp \left(\frac{1}{3} t\right)\left(\partial_{t}-\frac{1}{3} u \partial_{u}\right) .
$$

Case 2.3. $F(u)=a u+b, a, b-$ const, $a \neq 0$.

The invariance algebra of the equation

$$
L(L u)+L u=a u+b
$$


is a five-dimensional algebra $<P_{0}, P_{1}, Z_{1}, Z_{2}, Z_{3}>$, where

$$
Z_{1}=\left(x+\frac{b}{a} t\right) \partial_{x}+\left(u+\frac{b}{a}\right) \partial_{u},
$$

and two other operators depending on constant $a$ have the form

a) $a=-\frac{1}{4}$

$$
\begin{aligned}
& Z_{2}=\exp \left(-\frac{1}{2} t\right)\left(\partial_{x}-\frac{1}{2} \partial_{u}\right) \\
& Z_{3}=\exp \left(-\frac{1}{2} t\right)\left(t \partial_{x}+\left(1-\frac{1}{2} t\right) \partial_{u}\right),
\end{aligned}
$$

b) $a>-\frac{1}{4}, a \neq 0$

$$
\begin{aligned}
& Z_{2}=\exp (\alpha t)\left(\partial_{x}+\alpha \partial_{u}\right), \\
& Z_{3}=\exp (\beta t)\left(\partial_{x}+\beta \partial_{u}\right),
\end{aligned}
$$

where

$$
\alpha=\frac{-1-\sqrt{4 a+1}}{2}, \beta=\frac{-1+\sqrt{4 a+1}}{2}
$$

c) $a<-\frac{1}{4}$

$$
\begin{aligned}
& Z_{2}=\exp (\gamma t)\left(\sin \delta t \partial_{x}+(\gamma \sin \delta t+\delta \cos \delta t) \partial_{u}\right), \\
& Z_{3}=\exp (\gamma t)\left(\cos \delta t \partial_{x}+(\gamma \cos \delta t-\delta \sin \delta t) \partial_{u}\right),
\end{aligned}
$$

where

$$
\gamma=-\frac{1}{2}, \quad \delta=\frac{\sqrt{-(4 a+1)}}{2} .
$$

Case 2.4. $\quad F(u)=a, \quad a=$ const.

The invariance algebra of the equation

$$
L(L u)+L u=a
$$

is a five-dimensional algebra $<P_{0}, P_{1}, G, Q_{1}, Q_{2}>$, where

$$
\begin{aligned}
Q_{1} & =\left(x-\frac{a}{2} t^{2}\right) \partial_{x}+(u-a t) \partial_{u}, \\
Q_{2} & =\exp (-t)\left(\partial_{x}-\partial_{u}\right) .
\end{aligned}
$$

Thus, we have investigated the symmetry classifications of (4) and given all functions $F(u)$ under which the invariance algebra of (4) admits the extension. The new representations which can have an interesting physical interpretation have been obtained. The symmetry properties of (4) can be used for a symmetry reduction and construction of the solutions and for their generation by finite group transformations $[4,5,6]$.

In the case when Eq.(4) has the form

$$
L(L u)+\lambda L u=a, \quad a, \lambda-\mathrm{const}
$$


the change of variables

$$
\left\{\begin{array}{l}
t=\tau \\
x=\omega+u \tau \\
u=u
\end{array}\right.
$$

enables us to construct the general solution of (9). As a consequence of the change of variables (10), we obtain:

$$
\begin{aligned}
& L=\frac{\partial}{\partial t}+u \frac{\partial}{\partial x} \rightarrow \partial_{\tau}, \\
& L u=\frac{\partial u}{\partial t}+u \frac{\partial u}{\partial x} \rightarrow \frac{u_{\tau}}{1+\tau u_{\omega}} .
\end{aligned}
$$

After the change of variables, Eq.(9) has the form

$$
\partial_{\tau}\left(\frac{u_{\tau}}{1+\tau u_{\omega}}\right)+\lambda\left(\frac{u_{\tau}}{1+\tau u_{\omega}}\right)=a
$$

Integrating (11) once, we get a linear inhomogeneous partial defferential equation. Finding first integrals of the corresponding system of characteristic equations and performing the inverse change of variables, we find solutions of (9).

If $F(u) \neq$ const in (4), then this method does not lead to solutions. Below we give some classes of solutions of Eq.(9):

1. $L(L u)=0$

$$
\begin{aligned}
& 1.1 \quad x-u t+\frac{C}{2} t^{2}=\varphi(u-C t), \\
& 1.2 u \pm \ln (x-u t \mp t)=\varphi\left(t^{2}-(x-u t)^{2}\right), \\
& 1.3 u+\frac{t(x-u t)^{3}}{t^{2}(x-u t)^{2}-1}=\varphi\left(t^{2}-\frac{1}{(x-u t)^{2}}\right), \\
& 1.4 u=\varphi\left(\frac{x-u t}{\exp \left(t^{2}\right)}\right)-\frac{x-u t}{\exp \left(t^{2}\right)} \int \exp \left(t^{2}\right) d t .
\end{aligned}
$$

2. $L(L u)=a$

$$
x-u t+\frac{a}{3} t^{3}+\frac{C}{2} t^{2}=\varphi\left(u-\frac{a}{2} t^{2}-C t\right) .
$$

3. $L(L u)+L u=a$

$$
x-u t-C(t+1) \exp (-t)+\frac{a}{2} t^{2}=\varphi(u+C \exp (-t)-a t),
$$

$C=$ const, $\varphi$ is an arbitrary function.

Some results of this paper are published in [3]. 


\section{References}

[1] Fushchych W.I., New nonlinear equation for electromagnetic field having the velocity different from c, Dopovidi Akademii Nauk Ukrainy (Proceedings of the Academy of Sciences of Ukrana), 1992, N 1, $24-27$.

[2] Fushchych W., Symmetry analysis, in: Symmetry Analysis of Equations of Mathematical Physics, Kiev, Inst. of Math., 1992, 5-6.

[3] Fushchych W.,Boyko V.,Symmetry classification of the one-dimensional second order equation of hydrodynamical type, Preprint, Linköping University, Sweden, LiTH-MAT-R-95-19, 11p.

[4] Fushchych W., Shtelen W. and Serov N., Symmetry Analysis and Exact Solutions of Equations of Nonlinear Mathematical Physics, Dordrecht, Kluwer Academic Publishers, 1993, 436p.

[5] Olver P., Application of Lie Groups to Differential Equations, New York: Springer, 1986, 497p.

[6] Ovsyannikov L.V., Group Analysis of Differential Equations, Academic Press, New York, 1982, 400p. 